\title{
EXTRINSIC GEOMETRY SENSITIVE BOSONIC STRING THEORY
}

\author{
Al. R. Kavalov
}

Yerevan Physics Institute,

Alikhanyan Brothers St.2, Yerevan, 375036, Armenia, CIS

Abstract: A modified rigid string theory with infrared behaviour governed by a nontrivial fixed point is presented.

Some years ago the authors of the works [1-4] considered a string theory with the action containing, besides the usual Nambu term, also a term equal to the square of the extrinsic curvature tensor of the world surface:

$$
2 \alpha_{0} S_{0}=\sqrt{g} g^{\alpha \beta} g^{\gamma \delta} \nabla_{\alpha} \partial_{\gamma} X^{\mu} \nabla_{\beta} \partial_{\delta} X_{\mu}+\sqrt{g} \lambda^{\alpha \beta}\left(\partial_{\alpha} X^{\mu} \partial_{\beta} X_{\mu}-g_{\alpha \beta}\right) .
$$

Here $\alpha_{0}$ is a dimensionless coupling constant, and the Lagrange multiplier term just keeps the metric $g_{\alpha \beta}$ identical to the induced metric $\partial_{\alpha} X^{\mu} \partial_{\beta} X_{\mu}$. The role of $S_{0}$ is, classically, to prevent (energetically) the surface from bending and to lead the theory to a "smooth" phase with the string tension scaling near the critical point. Such a smooth strings are expected to provide a describtion of the universality classes of the 3-dimensional Ising model and 4-dimensional QCD [2], thus passing through the $c=1$ barrier of "conformal matter + Liouville" - type theories. They are also useful as the models of membranes, domain walls, etc. [1,3,4].

However, as was shown in the works [1-4], the coupling $\alpha_{0}$ turns out to be asymptotically free, which means that in the infrared the first term in $S_{0}$ is irrelevant, while in the second one the Lagrange multiplier field $\lambda^{\alpha \beta}$ developes an expectation value of the order of the cutoff, thus generating a nonzero effective string tension and driving the theory into familiar (and, from the point of view of $3 d$ Ising model and $4 d$ QCD, unacceptable) Liouville phase. The whole picture is analogous, apart from a numerical difference in the coefficient in the $\beta$-function, $d / 2$ instead of $d-2$, to what happens in non-linear sigma-models, where the quantum fluctuations generate a mass gap.

This situation can be avoided by generalising the action (1) in such a way that the $\beta$-function $\beta\left(\alpha_{0}\right)$ has a sero at some nonzero value of $\alpha_{0}$. Then, at the fixed point, the continuum theory will remain massless, with long-ranged correlations between the normal vectors of the surface. Such a generalisation will be described in the present letter. The details and some farther results will be presented elsewhere [8]. The action to be presented below is strongly suggested by the experience gained in attempts to describe the $3 d$ Ising model in terms of the fermionic strings [5,6], see also [7]. A basic object in our approach is a composite matrix field $\Omega \in S O(d)[5,6]$, constructed in the following way. 
Let $X^{\mu}(\xi)(\mu=1, \ldots, d)$ describe an embedding of some (closed, orientable) surface $\Sigma$ into $d$-dimensional Euclidean space. The tangent vectors of the surface will be denoted by $X_{\alpha}=\partial_{\alpha} X, \alpha=1,2$, and $h_{\alpha \beta}=X_{\alpha} X_{\beta}$ is the 2-dimensional metric tensor induced by the embedding. Introduce the zweibeins $e_{\alpha}^{a}(a=1,2)$, such that $e_{\alpha}^{a} e_{a \beta}=X_{\alpha} X_{\beta}$, then $X_{a}=e_{a}^{\alpha} X_{\alpha}$ are the orthonormal vectors tangent to the surface; let us introduce also $d-2$ orthonormal normals to the surface, $X_{i}(i=$ $1, \ldots, d-2)$, and denote the whole set of $d$ orthonormal $d$-dimensional vectors by $X_{m}^{\mu}=\left\{X_{\alpha}^{\mu}, X_{i}^{\mu}\right\}, m=1, \ldots, d$. Finally, consider a matrix field $\Omega$ representing the $S O(d)$ - rotation taking the basis set $X_{m}$ to some arbitrary constant orthonormal basis $X_{m}^{c}$.

Some comments are now in order. First, a theory with the action depending on $\Omega$ can not, in general, be considered a string theory, because of the ambiguity in the definition of $\Omega$ : it depends on the choice of the zweibeins $e_{\alpha}^{a}$ and of the normals $X_{i}$. To have a string theory one has to ensure an $S O(2) \times S O(d-2)$ gauge symmetry of the action, thus obtaining a theory with $\Omega$ taking values effectively in the Grassmanian $G_{2, d}=S O(d) / S O(2) \times S O(d-2)$. This can be done in a covariant way which we will not describe here; the net result is that after a gauge fixing one ends up with the following constraints to be imposed on $\Omega$ :

$$
\begin{aligned}
e_{a}^{\alpha} \nabla_{\bar{z}} e_{b \alpha} & =0 \\
X_{i}^{\mu} \nabla_{\bar{z}} X_{\mu j} & =0
\end{aligned}
$$

where the covariant derivative and the complex stucture must be taken compatible to the metric $h_{\alpha \beta}$.

The second comment is that for some embeddings the field $\Omega$ is singular. This happens when the surface has an open line of self-intersection. For $d \geq 4$ this singularities are not stable; however, for $d=3$ they are stable and play the most essential role in the string representation of $3 d$ Ising model $[5,6]$. In the local consideration presented here they can be ignored.

The third comment is that $\Omega$ can be taken in any representation of $S O(d)$. The one relevant for the $3 d$ Ising model is the spinor one $[5,6]$, but the construction works for a general case. In the present letter we will take $\Omega$ to be a $d \times d$ matrix

$$
\Omega_{m n}=X_{m}^{c} X_{n}
$$

Having this all in mind, let us write down the simplest possible action, depending on $\Omega$, namely that of the nonlinear sigma-model:

$$
4 \alpha_{0} S_{0}=\sqrt{h} h^{\alpha \beta} \operatorname{tr} \partial_{\alpha} \Omega \partial_{\beta} \Omega^{-1}
$$

Now substituting for $\Omega$ the expression (3) and using the conditions (2) one shows that, when rewritten in terms of the field $X$, the action (4) coinsides with the action (1). This observation suggests generalising the action (1) by adding a Wess-Zumino term to it. Namely, consider the action

$$
2 \alpha_{0} S=\sqrt{g} g^{\alpha \beta} \nabla_{\alpha} \partial^{\gamma} X \nabla_{\beta} \partial_{\gamma} X+\sqrt{g} \lambda^{\alpha \beta}\left(\partial_{\alpha} X \partial_{\beta} X-g_{\alpha \beta}\right)+\sigma / 3 \operatorname{tr}\left(\Omega^{-1} d \Omega\right)^{3} .
$$


In the last term of this expression an integration over some 3-dimensional manifold such that its boundary coinsides with the surface $\Sigma$ is understood, and $\Omega$ denotes now an arbitrary extension of the field (3) on this manifold. The couplings must be connected by Wess-Zumino quantisation condition

$$
\sigma / \alpha_{0}=i n / 4 \pi, \quad n \in Z
$$

Let us stress again, that the constraints (2), which have to be added to the action, arise as a result of the gauge fixing in some $S O(2) \times S O(d-2)$ - invariant theory, the explicit form of which is not important for us now.

In the rest of this letter I will show, in the one-loop approximation, that at some value of the coupling constant $a_{0}$ the action (5) describes a conformally invariant theory. For simplicity I will restrict myself to the case $d=3$; I will follow closely the line of calculations described by Polyakov in the work [2]. Suppose that the theory (5) is defined with the momentum space cutoff $\Omega$ and let us integrate over the fields with momenta satisfying the condition $\tilde{\Lambda} \leq|p| \leq \Lambda$. Denoting this "fast" fields by the superscript " 1 " on obtains for the quadratic term in the expansion of the conformal gauge $\left(g_{\alpha \beta}=\rho \delta_{\alpha \beta}\right)$ action in powers of the "fast" fields:

$$
S_{2}=X_{1} D X_{1}-2 X_{1} J-\lambda_{1}^{\alpha \alpha} \rho_{1}+\left(\partial^{2} X\right)^{2} \rho_{1}^{2}
$$

where

$$
\begin{aligned}
D^{\mu \nu} & =\delta^{\mu \nu}\left(\partial^{4}-\partial_{\alpha} \lambda^{\alpha \beta} \partial_{\beta}\right)+2 \sigma \partial_{\alpha} R_{\alpha \beta \gamma}^{\mu \nu} \partial_{\beta} \partial_{\gamma}, \\
R_{\alpha \beta \gamma}^{\mu \nu} & =\left[\partial_{\delta} X_{\sigma}^{\mu} X_{\delta}^{\nu} \delta_{\alpha \beta}+\partial_{\alpha} X_{\sigma}^{\mu} X_{\beta}^{\nu}\right] \varepsilon_{\sigma \gamma}, \\
J^{\mu} & =\partial_{\alpha} X_{\beta}^{\mu} \lambda_{1}^{\alpha \beta}+\partial^{2}\left(\partial^{2} X^{\mu}\right) \rho_{1}+ \\
& +\frac{\sigma}{2} \partial_{\alpha} n^{\mu}\left[3 H_{\beta \alpha} \varepsilon^{\beta \gamma}+i H_{\beta \delta} \varepsilon_{\beta \gamma} \varepsilon_{\alpha \delta}\right] \partial_{\gamma} \rho_{1},
\end{aligned}
$$

$H_{\alpha \beta}=n^{\mu} \partial_{\alpha} X_{\beta \mu}$ is the extrinsic curvature tensor, and we have put the background ("slow") value of $\rho$ equal to $1 . n^{\mu}$ is the (unique in $d=3$ ) unit vector normal to the surface. When deriving $S_{2}$ one has to take the variation of the matrix $\Omega$ keeping in mind the conditions (2).

One is left with a sequence of gaussian integrations which are performed quite straightforwardly, exept the one over $\lambda_{1}$ for which a decomposition suggested by Polyakov [2] is used:

$$
\begin{gathered}
\lambda^{\alpha \alpha}=\zeta, \\
\partial_{\alpha} \lambda^{\alpha \beta}=\partial^{2} f^{\beta} .
\end{gathered}
$$

As a result, in the leading logarithmic approximation the effective action turns out to be

$$
\begin{aligned}
2 \alpha_{0} S_{\text {eff }} & =\left[1-\frac{\alpha_{0}}{2 \pi} \log \left(\frac{\Lambda}{\tilde{\Lambda}}\right)+\frac{3 i \sigma \alpha_{0}}{8 \pi} \log \left(\frac{\Lambda}{\tilde{\Lambda}}\right)\right] \frac{\left(\partial^{2} X\right)^{2}}{\rho}+ \\
& +\frac{\sigma}{3} \operatorname{tr}\left(\Omega^{-1} d \Omega\right)^{3}+\lambda^{\alpha \beta} \partial_{\alpha} X \partial_{\beta} X-\left[1-\frac{\alpha_{0}}{4 \pi} \log \left(\frac{\Lambda}{\tilde{\Lambda}}\right)\right] \lambda^{\alpha \alpha} \rho .
\end{aligned}
$$


Performing an evident field renormalisation one finally obtains, using the quantisation condition (6), the following renormalisation equation for the coupling $\alpha_{0}$ :

$$
\tilde{\alpha}_{0}=\alpha_{0}-\frac{3 \alpha_{0}^{2}}{4 \pi}\left(1+\frac{n \alpha_{0}}{8 \pi}\right) \log \left(\frac{\Lambda}{\tilde{\Lambda}}\right) .
$$

From (8) we read off a $\beta$-function:

$$
\beta\left(\alpha_{0}\right)=\frac{-3 \alpha_{0}^{2}}{4 \pi}\left(1+\frac{n \alpha_{0}}{8 \pi}\right) .
$$

For $n=0$ this expression coinsides with the one found in the works [1-4] and describes an asymptotically free coupling. For $n \neq 0$, however, the $\beta$-function (9) has a sero at $\alpha_{0}=-8 \pi / n$. Since $\alpha$ must be positive, one has to take $n<0$ and we obtain an infrared stable fixed point.

The formula (9) is the main result of the present work. Let me add some comments to it. Though (9) was derived for $d=3$, an analogous result holds also for arbitrary $d$, with the coefficient in the $\beta$-function being $d / 2$ instead of the $d-2$ for the usual $G_{2, d}$ Wess-Zumino-Novikov-Witten model. This difference arises, as was stressed by Polyakov [2], as a result of restricting the field $\Omega$ by an integrability condition stating that $\Omega$ must be obtainable from a surface. The formula (9) shows that imposing such a restriction does not spoil the conformal properties of the Wess-Zumino-Novikov-Witten model, so that the model $(5,2)$ can be regarded as some reduction of it.

\section{Acknowledgements}

This work was reported on the Kiev conference on theoretical physics (June, 1992). The author would like to thank the organisers, and especially V. Shadura and S. Pakuliak, for support and for creation of the stimulating scientific athmosphere. The author is grateful to A. Belavin, An. Kavalov, A. Marshakov, A. Mironov,

R. Mkrtchyan, J. Petersen, J. Polchinski and A. Sedrakyan for the discussions of the ideas close to those reported in this work. Stimulating conversations with C. Bachas and J.L.F. Barbon are gratefully acknowledged.

\section{References}

[1] L. Peliti, S. Leibler, Phys. Rev. Lett. 54 (1985) 1609.

[2] A.M. Polyakov, Nucl. Phys. B 268 (1986) 406.

[3] H. Kleinert, Phys. Lett. 174B (1986) 335.

[4] D. Foerster, Phys. Lett. 114A (1986) 115.

[5] Al. Kavalov, A.Sedrakyan, Nucl. Phys. B 285 [FS19] (1987) 264.

[6] Al. Kavalov, A.Sedrakyan, Phys. Lett. 173B (1986) 449.

[7] Al. Kavalov, I. Kostov, A. Sedrakyan, Phys. Lett. 175B (1986) 331.

[8] Al.R. Kavalov, submitted to Nucl. Phys. B. 